\title{
Mass spectrometry analysis of HIV-I envelop proteins
}

\author{
A Galkin1, E Filinova², A Soloviev³, A Bychenko², N Polyakov³, V Nikiforov", \\ D Ulezko*3, E Gagarina $^{1}$ and N Samokhina ${ }^{2}$
}

\begin{abstract}
Address: ${ }^{1}$ Molecular Genetics Dept, Advanced Biomedical Research Laboratory, Moscow, Russia, 115446, ${ }^{2}$ Cellular Biology Dept, Advanced Biomedical Research Laboratory, Moscow, Russia, 115446, ${ }^{3}$ Protein Biochemistry Dept, Advanced Biomedical Research Laboratory, Moscow, Russia, 115446 and ${ }^{4}$ Physics Analysis Dept, Advanced Biomedical Research Laboratory, Moscow, Russia, 115446

* Corresponding author
\end{abstract}

from Frontiers of Retrovirology: Complex retroviruses, retroelements and their hosts Montpellier, France. 21-23 September 2009

Published: 24 September 2009

Retrovirology 2009, 6(Suppl 2):P37 doi:10.1 186/1742-4690-6-S2-P37

This abstract is available from: http://www.retrovirology.com/content/6/S2/P37

(C) 2009 Galkin et al; licensee BioMed Central Ltd.

\section{Background}

For many countries including Russia the development of preventive HIV vaccine became one of the crucial problems in the national survivorship. The purpose of the present study was to use phage display reverse panning (RP) technique for obtaining HIV-1 envelop proteins for LC-MS analysis of their major variability.

\section{Methods}

The naïve human ScFv antibody library expressed on the M13 surface was created using total RNA from lymphocytes of 20 HIV-1 subtype A infected Russian patients. Standard RT with random hexanucleotide oligos, cDNA matrix heavy and light repertoires PCR-amplification, ScFv genes PCR assembly and pCANTAB5E phagemid cloning were used. HIV-1-specific human ScFv libraries were selected after three rounds of biopanning with recombinant diagnostic and native HIV-1 peptides, HIV specificity was confirmed with ELISA and WB, recombinant phages were scanned with SPM contact mode (NanoWizard, JPK instruments). Ultrafiltration, ultracentrifugation, HIV-1-specific libraries immobilized on supermacroporous epoxy-activated cryogel RP (Protista Biotechnology) and SDS-PAGE, consequently, were used for viral proteins concentration. One-dimensional LCMS-MS Esquire6000Plus (Bruker Daltonics) tryptic cleavage virus peptide's identification was analyzed with Scaffold (UK) and PEAKS (Canada) software complexes and NCBI database.

\section{Results}

$64 \mathrm{ScFv}$ phage clones were analyzed in crisscross ELISA tests for HIV specificity. ScFv libraries enriched on diagnostic recombinant HIV peptides gp110 and gp160 provided higher ( $\geq 96$ percent, $\mathrm{p} \leq 0.003$ ) specificity to native HIV proteins from patients isolates than libraries after panning at laboratory U455 proteins. Therefore gp110gp160 phage-presented ScFv were immobilized at cryogel column and concentrated native HIV-1 subtype A proteins in RP successfully. Recombinant M13 has symmetric ScFv "head" structure at SPM images. Gp120 and gp41 peptide's LC-MS-MS identification was possible only in samples run through all concentration stages including reverse panning and was not possible in samples without RP. Best RP column eluate fractions were detected in WB. PEAKS complex can be used for HIV envelop peptides quantitative and variability analysis, Scaffold's potency is modest for it. Proteomics databases search revealed the predominance of HIV-1 subtype A Env, Pol and Gag tryptic peptides in native viral samples. The amount of the particular HIV-1 protein variant in the LC-MS sample correlated with the probability of its detection by this method.

\section{Conclusion}

RP concentration approach can be used for envelop variation's sequence identification of predominating native HIV-1 quasispecies, circulating in the bloodstream. We suppose RP technique and LC--MS-MS as crucial identification methods for HIV preventive vaccines creation. 